\section{TEORÍA DE SISTEMAS SOCIEDAD Y EDUCACIÓN}

THEORY OF SYSTEMS, SOCIETY AND EDUCATION

Enio Colagiàcomo* Ana Mendez de Garagozzo** UPEL-IPB

Recibido 11-02-08

\section{RESUMEN}

En el presente artículo refiere una investigación documental que tiene como propósito reflexionar críticamente sobre la Teoría General de Sistemas (TGS) como eje fundamental para accionar participativamente en la cotidianidad científica, tecnológica y educativa actual. Conocer esta Teorí han ha constituido remotos un recurso esencial para la alfabetización tecnológica se instituye como concepto fundamental para la comprensión de la complejidad humana, en virtud de poder complementar el método cuántico con la posibilidad operacional de incorporar cualidades incluso sentimientos en la producción de conocimiento. En la actualidad el principal objeto de estudio de la TGS es la gerencia de procesos en ámbitos sociales; por ello su conveniente aplicación en los contextos educativos, por cuanto viabiliza la conducción eficiente de éstos, siendo pertinente sobre todo el concepto autorregulador, autosustentador y autotransformador de los sistemas autopoiéticos desarrollansos por las comunistemas autopoiéticos desarrollados por las comunidades educativas para su consecuente mejora del servicio,
la gestión y la autonomía.

Descriptores: Teoría de sistemas, sociedad, educación, autopoiesis.

* Prof. Agregado en la UPEL-IPB. Adscrito al Dpto. de Educación Técnica, Programa Mecánica Industrial. Tuto de trabajos de grado. Investigador de la línea Tecnología, Producción y Prestación de Servicio, en áreas de la pedagogía y problemática de la Educación Técnica. Representante profesoral al Consejo Directivo UPEL-IPB. Doctorante de la Universidad Santa María en Ciencias de la Educación. e-mail: giacomontenio@hotmail.com

**Ingeniera en Electrónica, Magíster en Enseñanza de la Matemática y Doctora en Ciencias de la Educación. Ponente y conferencista nacional e internacional. Coordina la línea de investigación Saber Hacer Docente. Ponente y con Eocente. México, a decicon hón ext México, mención honorífica en investigación 2005, México. Es PPI. Tutora de trabajos de grado y tesis doctoral. e-mail: anamendezg@hotmail.com

\section{INTRODUCCIÓN}

"Os advierto que el todo es algo más que la suma de las partes” Aristóteles

Al hacer referencia a los artefactos, actualmente transformados en tecnofactos, ofrecidos por la sociedad postmoderna para la solución de los problemas cotidianos de trascendencia social, implícitamente también se alude a los procesos, las redes de procesos, las organizaciones y las innumerables acciones que participan en su constitución, así como las reacciones de indeterminada diversidad, generalmente no percibidas, incluso ni siquiera apreciadas por el propio actor social como usuario o beneficiario del quehacer científico, tecnológico, productivo y pedagógico, en los cuales el concepto de sistema es absolutamente inherente a todos ellos y su comprensión considerada esencial para la vida contemporánea.

De allí que el estudio formal de la Teoría General de Sistemas permite abordar con rigor científico gran cantidad de situaciones cuyo soporte epistémico deriva de conceptos subalternos, tales como: “insumos”, “procesos”, “resultados”, “realimentación” y lo relativo a flujos, materia, energía, información y autocontrol entre otros, los cuales se convierten en valiosas herramientas para el logro de objetivos de toda naturaleza, incluyendo el ámbito escolar en general, sobre todo en Educación Técnica y Tecnológica, donde las exigencias de precisión, interconexión, interrelación, seguridad y pertinencia social exigen a los maestros y estudiantes un mayor compromiso al interpretar los fundamentos que la convierten en un espacio de construcción de saberes practicables y de aplicaciones que coadyuven a una mejor calidad de vida de la sociedad. Por ello, la Teoría General de Sistemas (TGS) puede considerarse como conformante de la columna vertebral de la alfabetización tecnológica y estimablemente necesaria para comprenderla.

Se destaca que en el estudio de las ciencias sociales emergen situaciones propias de la complejidad humana, relativas a factores económicos, tecnológicos, naturales, culturales entre otros, imbricados en el quehacer cognitivo cotidiano, por ser el hombre objeto y sujeto cuando organiza, planifica, resuelve problemas y toma decisiones, bien sea en la industria, la gerencia de procesos, la economía, la defensa, la salud o en educación, en los cuales el enfoque de la TGS aborda la complejidad de los escenarios desde un estilo de pensamiento sin pretensiones reduccionistas, sustentado en la totalidad, fundamentado en 
una visión integral, abarcante e incluyente, articuladora de la tríada pensamientosentimiento-acción.

Lo antes señalado constituye un argumento reflexivo de considerable validez para la consecución de resultados favorables en el campo pedagógico, por cuanto en éste, el efecto sintagmático es esencial para conducir los procesos didácticos hacia los cambios de actitud de los discentes y docentes, al viabilizar la ineludible integración de paradigmas que exige el trabajo docente, que de acuerdo con el principio Aristotélico "el todo es mayor que la suma de las partes” se valida en las ciencias sociales y pedagógicas bajo la comprensión holística de los eventos, los procesos de construcción conceptual, las abstracciones y sus intrínsecas sinergias.

Esta indagación documental se significa en la reflexión crítica sobre la concepción sistémica que todo docente requiere conocer, para su mejor desempeño en las acciones cotidianas relacionadas con la gerencia de los procesos educativos, incluida la vida en el aula. Además, permitirá fundamentar los conocimientos teóricos para sustentar futuras investigaciones educativas bajo el paradigma sistémico, el cual es un tópico poco abordado como basamento epistémico, debido a la tradición reduccionista propia de la concepción mecanicista.

Así mismo, se pretenden confrontar críticamente las diversas posturas de los autores, en cuanto al tema en cuestión, para propiciar una síntesis articuladora, que aún sin pretensiones de construcción teórica cree espacios pedagógicos con sentido de completud, para reflexionar e impulsar un pensamiento sistémico auto-organizador de la acción educativa y su pertinencia en el entramado social de pertenencia.

En el desarrollo estructural de este artículo se ofrece una sucinta base conceptual genérica del paradigma sistémico, abordada en distintas etapas de la evolución del conocimiento humano por varios pensadores e investigadores, además se plantea el ámbito y las técnicas de gerencia sistémica como un referente clásico de la TGS, luego se presenta la teoría de los sistemas sociales y la subyacente imbricación lógica conceptual y operacional con la educación, para así, construir finalmente las reflexiones generadas por el análisis realizado por los investigadores.

\section{EL PARADIGMA SISTÉMICO}

Desde el pasado remoto, en el siglo IV y VIII A.C., Platón y Hesíodo, respectivamente, dejaron constancia de preocupaciones relacionadas con la comprensión de lo atinente al todo y la totalidad de los fenómenos estudiados; lo que implicó en lo sucesivo un trabajo sostenido en función de la búsqueda del conocimiento integral, la comprensión y aplicación del hecho científico para el avance de la humanidad; sobre todo, en dirección del fortalecimiento de su naturaleza gregaria y la solución a los problemas de convivencia y subsistencia; fundamentalmente cuando el espíritu indagatorio exigía dirigir la atención del investigador hacia el trabajo interdisciplinario, teniendo como referente empírico los complejos isomorfismos que caracterizan el comportamiento de los fenómenos biológicos y los del ámbito social.

En este sentido, Barón (2004) intenta explicar la incapacidad manifestada por las ciencias naturales para abordar esa complejidad cotidiana de la vida cognitiva humana, que deviene de los atributos propios del reduccionismo y sus consecuentes influencias de repetición mecanicista como paradigma referencial para la validación interna y externa del método científico, influido en muchos casos por una multivarianza difícil de controlar, debido a lo fortuito y la vulnerabilidad del método cuántico, propiciando así la opción de la implantación del paradigma de visión sistémica; lo que en la era postmoderna permite ver el fenómeno como génesis del análisis de la cualidad en el ámbito científico e investigativo.

Afirma el autor precitado, que en la década de los años cincuenta el biólogo Ludwin Von Bertalanffy presenta a la comunidad científica la Teoría General de Sistemas, con la cual pretendió configurar formalismos matemáticos que aún no se han consolidado en las esferas valorativas de la comunidad científica. No obstante, el pensamiento sistémico como paradigma referente goza de gran vigencia como recurso de vinculación formal entre las ciencias naturales y las sociales, dando fundamento al concepto de totalidad como suma no algebraica de los todos parciales, con capacidad orgánica de acción y reacción, generación de su propia sinergia, autoregulaciones y garantías holísticas de subsistencia y crecimiento progresivo sostenido o autodestrucción.

Lo antes indicado, como hecho científico novedoso impactó lo convencional del momento histórico; entre otros, al paradigma analítico, generando confrontaciones que aún mantienen cierta vigencia reflexiva en el campo educativo e investigativo y también grandes aportes a este ámbito de acción.

Por otra parte, Fuenmayor (2001) aboga por una teoría sistémico-interpretativa al visionar las organizaciones actuales. Su punto de partida es la deconstrucción argumentativa de la concepción tradicional de "lucha de poder" en las organizaciones y los postulados mecanicistas taylordianos de minimización de costos, optimización de la eficiencia y lucro, los cuales deben ser trascendidos para dar oportunidad a la emergencia de opciones que propongan nuevos modos de concebirlas, una renovada forma de pensar 
sobre ellas, donde prevalezca la virtud de las prácticas; es decir el desarrollo virtuoso en cuanto excelencia, cooperación, participación y no la acumulación de poder. El autor mencionado advierte que "Será en otro espacio y tiempo cuando se pueda agregar este contexto a la teoría sistémico-interpretativa de organizaciones” (p.108), aunque se destaca que el discurso actual de la gerencia sobre la forma de concebir las organizaciones ha cambiado, en él se incluye la calidad total, la reingeniería, la quinta disciplina, y la autopoiesis entre otras; esto alude al surgimiento de nuevas formas culturales en las organizaciones modernas, que coexisten con los modos tradicionales.

Esta visión sistémico-interpretativa permite crear un basamento comprensivo de los entendimientos y prospectos de cada factor influyente sobre la organización, además ofrece la posibilidad de ejercer una visión orquestada desde la perspectiva de las perspectivas que le otorga sentido a las acciones prácticas. De tal manera que en la institución educativa se puede construir, desde las relaciones de interpretación del devenir histórico de la cultura escolar, una similar comprensión, generada por las bases comunes donde se asientan sus fundamentos, como afirma el autor precitado.

Cabe considerar que Ander-Egg (2000) plantea que ante el parcelamiento que reduce y enceguece el conocimiento, se propone el abordaje interdisciplinario de la realidad, lo que requiere una actitud intelectual tendente a la interdisciplinariedad, para ello el enfoque sistémico articulado con una visión ecológica del mundo y del hombre, con sentido histórico y dialogante se constituye en un aporte fundamental coincidente entre pensadores.

\section{ÁMBITO Y TÉCNICAS DE GERENCIA SISTÉMICA}

En los procesos administrativos los principales objetos de estudio han sido los sistemas y sus elementos constituyentes, por ello, las personas encargadas de la administración de organizaciones han tenido que apropiarse de una importante y significativa área de conocimientos que se aproximen a una verdad que posibilite la realización de transferencias a la práctica, conducentes a la mejora en los resultados de los procesos gerenciales.

Según Melo (1994) la administración es un sistema cuyo ámbito está circunscrito al contexto social, además, es considerada un sistema abierto que permanentemente intercambia recursos con el medio; está conformado por personas que persiguen un fin común y al mismo tiempo constituyen el conjunto de interacciones humanas y técnicas para transformar los insumos en propuestas y resultados exitosos.
Por su parte, Bertalanffy (1976) explica que por lo general, las organizaciones vistas como un sistema se tipifican como sistemas artificiales, por cuanto son creados por el hombre y no están regidos por el patrón de nacimiento, madurez y muerte que caracteriza a los sistemas naturales. También puede considerarse a las organizaciones como sistemas abiertos; no obstante, no se deben suponer abiertos totalmente, tienen que tener límites que los protejan de los factores que puedan perjudicar al proceso, produciéndose así una selección de insumos que favorezcan su funcionamiento; por ello, se establece que los límites del sistema tienen un grado de porosidad variable que facilita la relación con el entorno y producen a la vez su propio equilibrio interno u homeóstasis, evitando así la entropía natural de todo sistema, constituyente de un efecto que tiende a la autodestrucción del mismo. Además, se debe citar la retroalimentación, la cual intenta incorporar modificaciones tendentes a optimizar los productos ya evaluados por el sistema.

La racionalización de los recursos y los objetivos de un sistema organizacional deben estar normados por una gama de técnicas aplicables en función de la naturaleza de la organización. Por ello, es necesario citar algunas técnicas que harán más factible comprender el enfoque sistémico y su aplicación en cada caso, según sea la naturaleza particular abordada.

Estas son conocidas como técnicas de administración y desarrollo organizacional (TADO), se dirigen básicamente a la solución de problemas de naturaleza fáctica y permiten al gerente tomar decisiones con la celeridad y la posibilidad que el mismo sistema permita, por cuanto debe estar presente un grado de predicción en su configuración teórica; por ello, no se puede suponer una técnica general en el análisis y solución de un problema específico, éste es quien requiere su adecuación, que dependerá del nivel y la complejidad al cual se enfrentan los gerentes, distinguiéndose solamente la ubicación del nivel objeto de estudio, ya sea estratégico, coordinador u operativo.

En tal sentido se cita la técnica de investigación de operaciones como uno de los enfoques sistémicos de investigación administrativa más usual, tanto en organismos públicos como privados, porque se ajusta al análisis para la toma de decisiones y hace énfasis en la construcción de modelos, considerando básicamente la operacionalización de enfoques como el análisis de riesgo y la teoría de la preferencia, signado respectivamente por la probabilidad matemática y el grado de riesgo que están dispuestos a asumir los gerentes.

En relación a la técnicas de control de gestión, Colagiácomo (2000) expone que es usual encontrar técnicas como el análisis de redes y hechos (PERT), las cuales relacionan los acontecimientos con el tiempo, y la del presupuesto que es una de las prácticas más 
antiguas fundamentada sobre la base de la formulación de planes en términos numéricos, además se consideran los presupuestos por programas que representan la expresión más moderna de la planeación corporativa con un enfoque contingente y la filosofía de calidad total como un paradigma referente holístico de ámbito tecnológico contemporáneo.

Asimismo, Dechtian, Jiménez, Marín y Rodríguez (1986) afirman que en la etapa organizativa de los procesos se practican técnicas como: (a) la simplificación del trabajo, la cual consiste en lograr fórmulas para ahorrar esfuerzos humanos, materiales, financieros, de máquinas y espacio; (b) el estudio de movimiento y el tiempo, donde se hace énfasis en cronometrar el tiempo normal requerido para ejecutar tareas y el movimiento indispensable para el logro de las mismas; y (c) los sistemas administrativos que constituyen un cuerpo de criterios y procedimientos lógicos dirigidos a la delineación de estructuras organizativas y de información entre unidades orgánicas, con el objeto de integrar mediante procesos de formalización todas las acciones relativas a la planificación, las decisiones y los controles; mediante esta técnica se delimitan con precisión las unidades orgánicas, las funciones, los cargos, las tareas que se repiten y el procesamiento de la información.

Los autores precitados coinciden al señalar la formalización, como técnica fundamental operativa de los procedimientos sistémicos gerenciales que recopila todas las actividades del nivel operativo y puede generar información que se describe detalladamente mediante símbolos que producirán efectividad en el control, coordinación y dirección de la ejecución de un proceso. Asimismo, facilitará la toma de decisiones de forma oportuna y rápida, por cuanto el proceso de formalización guiará al gerente con precisión hacia los organigramas, como expresión gráfica de la estructura, hacia los flujogramas como representación gráfica de los procedimientos administrativos y hacia los manuales, siendo ésta, la expresión descriptiva de la estructura y los procedimientos, los cuales soportarán mediante sus propios controles el éxito previsto en la formalización operativa del trabajo.

Por ello, en el ámbito organizacional los manuales constituyen un excelente aliado para mantener el equilibrio y la autorregulación sistémica prescrita por la teoría de sistemas y en consecuencia se yergue como un apoyo fundamental, neguentrópico, para el mantenimiento preventivo y correctivo de la moderna gerencia de procesos.

\section{LA TEORÍA DE LOS SISTEMAS SOCIALES}

Los sistemas no naturales, que interesan al investigador de las ciencias sociales se inscriben necesariamente en ese ámbito, el de los sistemas sociales, los cuales, por extensión son admitidos en los mismos términos genéricos pautados por la teoría de sistemas cuando estudia los sistemas naturales, con la necesaria previsión de los efectos que la naturaleza humana imprime a este paradigma.

Según la acepción práctica de la gerencia moderna, los sistemas pueden ser cerrados o abiertos. Según Martínez (1999) pueden ser lineales o no-lineales; los primeros no admiten intercambio alguno, todo ocurre dentro del sistema, nada tiene contacto con su medio interior, los segundos son los sistemas más pertinentes a las ciencias sociales, cuya utilidad ha sido múltiple y variada en las diferentes investigaciones realizadas en las últimas décadas, demostrando ser excelentes aliados para esos fines, en consecuencia, puede afirmarse que la teoría de sistema es un esencial apoyo para el investigador social.

Del origen etimológico-conceptual del vocablo sistema se ha derivado, para las ciencias sociales, el término "sistemas sociales", de quien se debe hacer referencia obligatoria por su enorme utilidad como herramienta conceptual y de análisis, cuyo antecedente más reciente, es el término “organización social” utilizado medio siglo antes de la postmodernidad para caracterizar a las agrupaciones humanas. De allí, la existencia de una serie de fenómenos colectivos notablemente interdependientes e interactuantes que al final constituyen la sociedad humana de forma integral, cuyo carácter puede ser micro o macro social, con la peculiar característica que permite visualizar un todo heterogéneo humano en perfecto orden interactivo recurrente, que es lo que la teoría de sistemas admite como visión holística de esa sociedad humana.

En consecuencia, como producto de la interacción humana, han ocurrido eventos que de alguna forma marcan la naturaleza del hombre, entre ellos, como se sabe, los conflictos bélicos han sido marco de referencia en múltiples acontecimientos, los cuales han trascendido la era moderna y lamentablemente mantendrán su vigencia posiblemente por tiempo indefinido.

En el desarrollo del trascendente hecho precitado, el hombre organizó sus saberes en función jerárquica de esos acontecimientos sociales de interés gregario e individual, de manera que, por ejemplo, las operaciones bélicas al pluralizarse, permitieran un importante grado de practicidad; acuñándose así un vocablo clave en el lenguaje sistémico actual: la “operacionalización”, cuya acepción expresa, en la postmodernidad, el soporte básico del acto procedimental sistémico.

Interpretando a Austin (2002), se entiende por esquema procedimental la secuencia preestablecida de manera lógica, ordenada y sistemática que permite desarrollar la ejecución de la acción como parte esencial de la operacionalización del proceso previsto, es la acción medular requerida para dar cumplimiento al logro de los objetivos establecidos. 
Asimismo, como complemento al concepto de la expresión “operacionalización” es pertinente aclarar que en el lenguaje sistémico se entiende como una expresión derivada de la rutina de la segunda guerra mundial, en la cual la incertidumbre de ocurrencia de eventos con alta probabilidad, complejidad logística y cantidad de individuos comprometidos, forzó un desarrollo creativo hacia la conformación de una metodología que involucró el análisis estratégico de sistemas interdependientes, los cuales para el desarrollo de las acciones bélicas representó una alternativa que culminó como una esencial herramienta para el éxito de las misiones impulsadas en ese momento. Para ilustrarlo, se puede referenciar la conocida metáfora marcial de la "invasión del día D a la hora H”, como una expresión operacional de una planificación estratégica contingencial de puntuales previsiones.

De allí que las organizaciones de producción y servicios de la postguerra o de la modernidad, incorporaron la revolucionaria disciplina planificativa como operación de sistema; según la cual la interdisciplinaridad con clara interrelación organizada de lo heterogéneo y la intencional participación del planificador, establecían visionariamente el éxito de las misiones en dirección de sus intereses como lo estableció Bertalanffy (ob.cit.) en sus estudios precursores de la TGS, al consolidar su sueño relativo a la posibilidad de desvincular la teoría de sistemas de la biología y universalizarla hacia todos los saberes, incluso las ciencias sociales, como ocurre en la actualidad con los sistemas sociales en general y los sistemas educativos en particular, entre otros.

Por su parte, Bunge (2000) establece como punto inicial del pensamiento sistémico un sistemismo ontológico, que deviene de un realismo práctico, donde el mundo es "un sistema de sistemas", es decir un suprasistema; parte de la premisa que los sistemas son complejos, interactuantes e interrelacionados entre sí, lo que conlleva una naturaleza del sistema social humano conformado por las personas y sus vínculos bien sean políticos, económicos, biológicos o culturales entre otros.

Este autor en su sistemismo ontológico distingue que un sistema particular se describe o debe contener en su composición o partes que lo conforman, el entorno como la congregación de elementos externos que influyen en el sistema o que resultan influidos por él, la estructura referida a los vínculos que instauran los componentes de un sistema y el mecanismo referente a los proceso que le dan vida, propician el cambio, la estabilidad o la desintegración.

Es de hacer notar que la postura de Bunge conlleva una mirada realista que confluye con una visión positivista de pensar y actuar en el mundo. En la actualidad los investigadores sociales muestran un importante grado de escepticismo respecto del epistema referido, negando su identificación con el realismo práctico, tendente al pragmatismo del sistemismo que planteó este autor, por cuanto la acción socioeducativa del docente actual no se reduce a un problema de fines y medios; esta sería una mirada positivista de entender la educación; en tal sentido la postura sistémica propuesta debe generar reflexiones y acciones autorreguladoras que ordenen la realidad para sí e involucren más la naturaleza humana.

De acuerdo a lo expuesto se asume que la visión holística e integral de la sociedad humana excluye la postura atomista que fragmenta y reduce, establece la premisa que el todo es mayor que -o al menos, diferente que- las partes. Asimismo, la idea de totalidad es una propiedad inherente a los sistemas, por cuanto según López, Parada y Simonetti (1995) se comportan como un todo indivisible y coherente, interrelacionando sus partes de manera que al afectarse una de ellas se afecta sensiblemente todo el sistema, en consecuencia una característica fundamental de una totalidad social es la sinergia.

En este sentido, interpretando a Waztalvick, Beavín y Jackson (1995) el concepto de sinergia se refiere a que la totalidad del fenómeno estudiado no es semánticamente igual a la suma cuántica de sus partes, sino que al analizarlo se debe apreciar a las partes en el ámbito de la complejidad organizativa del mismo y a sus consecuentes resultados. Al respecto, Martínez (ob.cit.) cuando se refiere a la estructura de los sistemas sociales, expresa que ésta no solo determina una configuración física de elementos, sino que son elementos de significados con una dinámica propia, siendo esta característica exclusiva de los sistemas no lineales, en los cuales:

(...).cada "parte", al formar una nueva realidad, toma en sí misma algo de la sustancia de las otras, cede algo de sí misma y en definitiva, queda modificada. Esto es lo que le sucede a cada ser humano al entrar a formar parte de un grupo social. (ob.cit., p.86)

Asimismo, Austin (ob.cit.) al explicar las ideas relativas a la visión holística de la sociedad humana, ilustra el concepto de sinergia de los sistemas sociales exponiendo que siempre son sinérgicos, como ocurre con una organización educativa, la cual es un subsistema social, donde una de sus partes no podrá producir en pequeño el producto final de la escuela; como es la formación del ciudadano para el desempeño social pleno.

Una de las propiedades más relevantes y útiles de los sistemas precitados es que no se reproducen en modelos vacíos o aislados, por tener un entorno 
conformado por otros sistemas, subsistemas, factores o fenómenos que generalmente influyen o son influenciados por el sistema referencial, dependiendo del grado de permeabilidad de sus límites. Con respecto a ello, Luhmann (citado por Austin, (ob.cit.)), al intentar explicar tal influencia expone:

Los sistemas no solo se orientan ocasionalmente o por adaptación hacia su entorno, sino de manera estructural, y no podrán existir sin el entorno. Se constituyen y se mantienen a través de la producción y el mantenimiento de una diferencia con respecto al entorno, y utilizan sus límites para regular esta diferencia. (p.50)

Luego, se concluye que los sistemas sociales se relacionan con su entorno de manera interactiva y simbiótica, al influir y ser influenciados en el ámbito del complejo sistema macrosocial. Al respecto, relacionando los sistemas sociales con los elementos de entrada y salida, o de insumos y productos interactuando con el entorno, Garvía (1998) expresa que un sistema cerrado es aquel que "no recibiendo imputs del exterior, tiende a la entropía, es decir, al agotamiento y caos interno. Por el contrario un sistema abierto en el que recibiendo energías o imputs del exterior es capaz de renovarse; entonces se dice que tiene entropía negativa” (p.97), también conocida en el campo de la teoría de sistemas como neguentopía. Denotándose que el equilibrio es para los sistemas abiertos la categoría dominante, con una capacidad dinámica de autorregulación para los outputs y la estabilidad de su vitalidad.

Otra propiedad de interés o capacidad presente en los sistemas abiertos, señalado por Rodríguez y Arnold (1990), es la retroalimentación, ésta es soporte esencial de la cibernética, definida como una ciencia de la acción, capaz de autocorregirse continuamente hasta obtener un objetivo; aspecto éste, en el cual centra su atención de manera especial el ámbito organizacional, las acciones de control de procesos y más recientemente la inteligencia artificial. En el caso de los sistemas sociales se refiere a la capacidad que tienen para mantener estable su dirección o finalidad sistémica; incluso llegar a su autoevaluación; los cuales también son soportes fundamentales para la comprensión del concepto de "autopoiesis".

Este constructo -autopoiesis- ampliamente estudiado en el campo de las ciencias sociales desde la década de los años setenta, después de derrumbarse la armadura cognitiva del funcionalismo, sustentó el estudio de los sistemas dando explicación a la autonomía o capacidad de autorregulación de ellos, independientemente de quién o qué les haya dado vida inicial.
En la teoría de sistemas la autopoiesis se fundamenta en principios esenciales como la ya mencionada retroalimentación, lo cual implica que los sistemas abiertos, como los sociales, contienen formas operativas internas que les permiten sostener la dirección correcta o finalidad sistémica, imprimiéndoles una suerte de régimen automático; ejemplo de ello podría ser: las distintas instituciones que pueden presionar o intentar corregir la dirección que sigue el conjunto social implicado en un hecho determinado, cumpliéndose según Rodríguez y otros (ob.cit.), el principio de la equifinidad, implicante de la capacidad que pueden tener los sistemas para llegar a un mismo fin partiendo de inicios distintos. Esta premisa, ya citada como el principio de la cibernética sistémica, se considera el segundo principio esencial de la autopoiesis.

En el mismo sentido, los autores precitados hacen referencia a la homeóstasis como el tercer principio esencial requerido para que en un sistema se propicie la autopoiesis. La homeóstasis describe la tendencia de los sistemas -sobre todo los naturales- a mantener factores críticos como la temperatura de un ser vivo, densidad poblacional y otros, dentro de un rango críticamente limitado o autolimitado.

En general, se puede afirmar que en el ámbito de las ciencias sociales la homeóstasis podría ser comparable con la capacidad sistémica de soportar en forma natural, hasta un límite, la estructura sistémica que se ha establecido o le han establecido para su estabilidad operativa; lo opuesto, actuará de forma que el sistema pueda entrar en cambios profundos de desintegración (entropía) que lo conduciría indefectiblemente hacia nuevas finalidades, lo que es muy significativo y elocuente para explicar los "cambios sociales”. Es así como este efecto indica que la comunicación interna del sistema no opera correctamente, las fuerzas entrópicas se apoderan de él, luego vence la tendencia, también natural, hacia el desorden y el caos. En consecuencia se rompe el límite admitido por la homeóstasis del sistema primario e irremediablemente inicia su auto extinción. Para que esto sea contrarestado, el sistema deberá fortalecer su capacidad de autopoiética, mediante la cual cumplirá el cometido sistémico de mantenerse operativo.

Al respecto, Maturana (1990) expone un concepto de sistema con una connotación dinámica y en constante evolución, aportando un renovado significado de sistema social, el cual emerge como resultado de la articulación social a través del lenguaje y de toda forma de comunicación o transferencia de información en una sociedad, desde la cotidianidad, así mismo se apoya en el concepto autopoiético para distinguir los sistemas sociales que se autorregulan desde la comunicación consigo mismo y con el medio para autoproducirse.

En tal sentido, la capacidad de autoproducirse mantiene estrechamente vinculado el sistema con el entorno, al intercambiar información, insumos y los elementos que lo 
“mantengan vivo”, activo y regulado en su función originaria como sistema. Es así que una sociedad vista como un sistema social requiere mantener su estructura de relaciones y redes internas que le dan autonomía o sentido a su existencia como sociedad particular, además debe tener capacidad para interactuar y responder a las perturbaciones externas que lo pudieran desequilibrar, a fin de adaptarse, sobrevivir y producir sus componentes, así como los procesos reguladores que lo identifican. En corolario, estas afirmaciones conllevan la idea de autoría de sus operaciones, lo que le caracterizará como subsistemas con porosidad para el intercambio de información e insumos con el suprasistema.

Actualmente no se percibe un sistema social desde la postura estructuralista, referido a las relaciones o estructuras constitutivas, sino que se comprende el proceso de su autoconstitución a través de la construcción de sus elementos y la apropiación de los estados conformantes y particulares para su autoorganización y autoproducción distintiva, a fin de mantener una demarcación o límite con el entorno que le dé oportunidad a su autosostenibilidad.

\section{La teoría de sistemas y la educación.}

Los sistemas que interesan al educador se circunscriben al ámbito de los sistemas sociales, los cuales, por extensión son admitidos en los mismos términos pautados por la teoría de sistemas. En razón de ello en el ámbito escolar es costumbre referirse al sistema educativo, pero esta expresión no alude a una comprensión y utilización consciente, reflexiva, del concepto, como fundamento paradigmático esencial interactuante entre los suprasistemas y los subsistemas conexos.

Asimismo, el principio de recursividad, posibilita visionar los distintos niveles de educación como una suerte de sistemas unitarios articulados; además, permite ver al sistema educativo nacional como contenedor de sistemas o subsistemas regionales, los cuales también contienen sistemas educativos menores, pasando por la unidad educativa y otros subsistemas intervinientes. Evidenciándose así que los sistemas educativos por ser subsistemas subalternos de un suprasistema en activa interacción, guardan una determinante semejanza que los caracteriza genéricamente como sistemas subalternos con una "genética sistémica" ineludible.

Estas propiedades individuales, con énfasis en la recursividad, facilitan la posibilidad de considerar los sistemas educativos como unitarios, de modo que el sistema educativo nacional contiene en sí, los regionales, estadales, municipales, distritales, parroquiales y locales, los cuales pueden ser disgregados mucho más, por cuanto cada una de las unidades operativas de una institución es susceptible de similares características al distinguirse en la escuela, por ejemplo, un sistema directivo y tantos como la naturaleza de la organización requiera para su actividad cotidiana.

En general, cualquiera de estos suprasistemas o subsistemas son susceptibles de análisis desde sus corrientes de entrada -insumos- y lo que surge de sí, hacia su entorno producto- pasando por los múltiples procesos internos más básicos como son: (a) el proceso de socialización que permite la autopoiesis del sistema, (b) la institucionalización en la cual están presente las fuerzas entrópicas, (c) el proceso de control y cambio social, donde actúan los elementos neguentrópicos y la homeóstasis que viabilizan la adaptación, así como el ajuste del sistema y (d) el proceso cultural, una suerte de integración de todos los todos -procesos- que de manera selectiva, simultanea, integral e indefinida de interacción y comunicación con el entorno, ambiente o contexto del suprasistema escolar, conforman la totalidad sistémica que en este particular caso describiría una parcialidad social.

Es por ello, que la escuela siempre tenderá a asumir comportamientos semejantes a los descritos para conjuntos de seres humanos en el ámbitos de la producción y los servicios; sobre todo en el nivel o modalidad escolar de formación profesional técnica y tecnológica, en los cuales la sinergia acciona sobre los productos finales incluso en forma no deliberada en virtud de que ninguna de las partes constitutivas del sistema formativo podrá producir por separado el resultado integral esperado, que en el caso educativo pudiera ser el conocimiento general y las competencias específicas con su correspondiente dosis de accionar práctico materializado en pericias y habilidades kinestésicas propias o específicas de la acción laboral, incluso actitudes y valores técnicos, tecnológicos y sociales.

En referencia a ello, es pertinente referir la percepción que manifiesta Ander-Egg (1995), como una valiosa opinión en el campo pedagógico, cuando plantea sus críticas opiniones sobre la visión sistémica y las consecuencias perfectibles en el ámbito educativo, al acotar que el enfoque sistémico

... se esfuerza en conseguir la eficacia del sistema total, más que por mejorar la eficiencia de las partes o subsistemas sin tener suficientemente en cuenta la interdependencia e interconexión de cada una de las partes que interactúan. Estas consideraciones no solo son válidas para aplicar en una institución docente, sino también para todo el sistema educativo. (No) afirmamos que sea la única manera significativa de estudiar la administración y las organizaciones. En este momento, nos parece la más significativa, habida cuenta del desarrollo actual de la ciencia y las transformaciones que se han ido dando en las organizaciones. (pp. 72 -73) 
En el ámbito cognitivo propiamente dicho, también se conocen hallazgos relevantes que confirman su conformación operacional y sistémica, sin que esto signifique rigidez, dogma o fórmula preestablecida para predecir las respuestas humanas en cuanto a los epistemas cognitivos. Al respecto, Pozo (1997) explica el comportamiento de las entidades mentales, los estados, procesos y la disposición de naturaleza mental; concibe esta actividad como una tarea orientada a procesar la información inteligente, la cual cumple varias fases esenciales como: (a) la entrada, -adquisición sensoperceptiva o percepción(b) la elaboración, -trasformación de la información- y (c) las salidas -producción de nueva información, costructos y aprendizajes significativos- como productos.

Ruiz (1997) reafirma este epistema sistémico de la actividad mental y la complementa en su aporte al sistema cognitivo humano ( $\mathrm{SCH}$ ), el cual concibe como un conjunto de operaciones y estrategias utilizadas por el sujeto para: (a) adquirir, retener, recordar y transferir -o procesar- información de las nuevas situaciones; y (b) para autorregular su propio funcionamiento cognitivo, lo que en la práctica sistémica se denomina autopóiesis. En la actualidad significa la auto formación continua que la didáctica centrada en procesos pretende instaurar como innovación pedagógica contemporánea.

En el mismo sentido, Barrera (2005) opina que en holística se considera que "el uno es complejo y la realidad es integral”, habida cuenta del carácter sinérgico de los eventos; surgiendo en consecuencia, el efecto sintagmático, lo cual constituye una integración de paradigmas que de acuerdo con el principio Aristotélico “el todo es mayor que la suma de las partes” se valida en las ciencias sociales y sobre todo en las pedagógicas bajo la comprensión holística y sintagmática de eventos, procesos de construcción conceptual, abstracciones y su sinergia contextual creciente.

Al respecto, este carácter sinérgico de los eventos y la dimensión integral de la realidad vienen instituidos desde el entendimiento de la realidad social como construcción de las intersubjetividades, donde la subjetividad se piensa a partir de la articulación sistémica con el contexto social para comprender y explicitar el desenvolvimiento y el desarrollo humano, como expresa Correa (2004), porque el mundo se percibe y se actúa en él direccionado por el entramado conformado por los intereses, los saberes, las emociones, las expectativas, las creencias que finalmente concretan en su sedimentación la subjetividad, es el eje conectivo entre lo social-cultural y la apropiación de la vida cotidiana de ese contexto, sus actividades y experiencias, lo que redundará en un ciudadano integral con competencias para vivir pro-activamente en sociedad y para la sociedad.

\section{REFLEXIONES FINALES}

Una mirada sistémica fenomenológica involucra sin duda la formación pedagógica de los actores, también sistémica, que conlleva la proyección consciente y la aplicación de una gama de acciones gerenciales tendentes a la búsqueda del éxito, como ha ocurrido en otros ámbitos del desenvolvimiento humano de la era moderna y posmoderna. De esta manera, al agotarse los modelos propuestos desde la sociedad industrial se instauran novedosos esquemas y propuestas que emergen a partir de la comprensión del accionar de la sociedad del conocimiento. Para ello se requiere una formación sistémica humanista cuyo sentido se oriente a mejorar el saber hacer sin menguar el ser; lo que debe conducir al logro de la eficacia de los procesos fundamentada en el cooperativismo, las habilidades comunicacionales, intelectuales, afectivas y una cultura ecológica erguida sobre la base de competencias básicas antes que el parcelamiento disciplinar.

Luego, un nuevo y pertinente sentido educativo se estima significativo para el docente, que aborde la tensa relación entre subjetividad, instituciones y sistemas, como afirma Correa (2004) "La subjetividad requiere de la eficiencia y creatividad de los sistemas autorregulables, tanto como estos requieren de la orientación subjetiva y valorativa para ser social y humanamente sustentables” (p.141), es una relación dialógica y mutuamente consecuente con las necesidades de cambio, como una construcción social patrocinada desde las instituciones educativas, emergente del entramado sistémico, para abordar la formación desde la unidualidad del ser biológico y cultural, para autoadministrarse con estrategias de pensamiento crítico, creativo y flexible, a fin de interpretar los cambios de paradigmas y manejar los avances tecnológicos con sentido social, solventando las desigualdades.

Para ello se requiere instrumentar reformas educativas en función de no quedar al margen de las exigencias de la economía, los desenvolvimientos mundiales y las nuevas formas de organización. En general, se comprende como un sistema capaz de adecuarse a los cambios y autoconstituirse críticamente, para dar respuestas asertivas que coadyuven a mejorar las condiciones de existencia de la sociedad.

En ese sentido interviene la TGS como sustento epistémico que pretende contribuir con la formación integral de los actores escolares, como seres humanos capaces de autodeterminarse y autoregularse desde la comprensión de la relación entre la subjetividad y las condiciones externas del medio social, identificado con su lugar en la sociedad y el papel histórico que en ella tiene, emparentado con los otros desde la comprensión de la naturaleza del nosotros para desarrollar lazos cooperativos, comprensivos y solidarios. 
De allí que la visión sistémica viabiliza abordar los subsistemas formativos como sistemas autocorrectivos que pueden ser informados y enriquecidos por proyectos transversales, con acciones practicables en sistemas subalternos como los proyectos pedagógicos. Al respecto, Peinado y Rodríguez (2007) afirman que la participación de los actores de la comunidad académica buscan dinámica y sistemáticamente desarrollar el conocimiento, las habilidades, destrezas, actitudes y valores, mediante intencionadas estrategias sugeridas por el currículo institucional, lo cual emplaza al docente como gerente educativo a realizar una gestión humanista conducente a una formación integral, mediante la propuesta de escenarios educativos que hagan de la ética y la estética el sostén de la formación, a través de la participación, la problematización, el diálogo y la coherencia, para fortalecer la dimensión moral de los ciudadanos desde el desarrollo y ejercicio de los valores más trascendentales, para dar respuestas asertivas que coadyuven a mejorar las condiciones de existencia, convivencia y democracia.

En síntesis la TGS aún cuando no se presenta deliberadamente fundamental ha promovido los soportes para la gestión de nuevos paradigmas pedagógicos identificados como "autoestructurantes", en los cuales el conocimiento debe necesariamente emerger “...en la medida que éste repose en el testimonio de la experiencia” (De Zubiría, 2006, p.112) siendo esta precisión una forma manifiesta de autocontrol, autorregulación y autoevolución; conceptos muy vinculados al consecuente proceso autopoietico propio de la realimentación sistémica de procesos; por ello la actual sociedad visiona una escuela que promueva condiciones de facilitación, manipulación y experimentación por parte de los actores, esperándose como resultados una sociedad más humana, productiva y ética; que valore el sentido social del conocimiento, lo que en suma soporta el paradigma de la pedagogía activa o de la acción, que se contrapone a la pedagogía tradicional.

La TGS se constituye entonces en un puente que une: el medio, la familia, la escuela, los docentes, padres, alumnos y egresados como subsistemas sociales de fines convergentes; utilizando para ello las mismas leyes que viabilizan: (a) el fortalecimiento de la vida como la actitud individual y colectiva para el mantenimiento de la especie en el ámbito cognitivo y social; (b) el claro descubrimiento de cuál es el lugar dentro del sistema que a cada sujeto le corresponde y el rol que debe asumir; (c) contextualizar el espacio y el tiempo bajo el paradigma de la recursividad autoregulante, a fin de ordenarse para sí ante las eventualidades de las incertidumbres, en razón de las metas sociales y cognitivas alcanzadas sobre la base de la reingenierías social y cognitiva requerida para ajustar los insumos y procesos y (d) reeditar al sujeto como agente de cambio social.
Finalmente, es pertinente señalar que la TGS, por medio de la pedagogía sistémica, no es la panacea para generar todas las soluciones de los problemas de los procesos educativos; siempre quedarán asuntos por resolver, no obstante, este enfoque podrá apuntalar la sistematización de los contenidos curriculares cognitivos, afectivos y procedimentales para facilitar la mediación educativa y fortalecer las competencias, como también las estrategias didácticas a utilizar; y el cómo abordarlas dependerá del nivel educativo en el cual se pretenda implantar.

\section{REFERENCIAS}

Ander-Egg, E. (1995). La planificación Educativa. Buenos Aires: Lumen.

Ander-Egg, E. (2000).Interdisciplinariedad en Educación. Buenos Aires: Magisterio

Aron, A. y Milicic, P. (1994). Vivir con otros. Programa de Desarrollo de Habilidades Sociales. Argentina: Universitaria.

Austín, T. “Teoría de Sistema y Sociedad” La página del Profe. Disponible: http://www.geocities.com/tomaustin_d/soc/sistema.htm [consulta: 2002, 0ct. 13].

Barón, M. (2004). Enseñar y Aprender Tecnología: Propuesta didáctica desde la teoría de sistemas. Buenos Aires: Novedades Educativas.

Barrera, F. (2005.). El Intelectual y los Modelos Epistémicos. Fundación Servicios y Proyecciones para América Latina. SYPAL

Bertalanffy, L. (1976). Teoría General de Sistemas Madrid: Fondo de cultura económica.

Bunge, M. (2000). La investigación científica. Su estrategia y su filosofía. México: Siglo XXI Edit.

Colagiácomo, E. (2000). Manual de Funciones y Procedimientos para la Tutoría del programa de Pasantías de la Escuela Técnica Industrial "Pedro León Torres" de Barquisimeto. Trabajo de grado no publicado. Barquisimeto: UPEL-IPB.

Correa de Molina, C. (2004). Currículo Dialógico, Sistémico e Interdisciplinar. Colombia: Editorial Magisterio 
Dechtian, E., Jiménez, P y Marín, E. y Rodríguez, W. (1986). El Proceso Organizativo en la empresa Droguería Dreyer Viloria C.A. de Barquisimeto. Trabajo de grado no publicado. Mimeografiado, Escuela de Administración y Contaduría. UCLA.

De Zubiría, J. (2006). Los Modelos Pedagógicos. Hacia una pedagogía dialogante. $2^{\text {a }}$ Edic. Bogotá: Magisterio.

Fuenmayor, R. (2001). Interpretando Organizaciones. Una teoría sistémico interpretativa de organizaciones. Venezuela: Universidad de los Andes.

Garvía, R. (1998). Conceptos fundamentales de la sociología. Editorial Alianza.

López, A., Parada, A. y Simonetti, F. (1995). Introducción a la Psicología de la Comunicación. Chile. Edit. Universidad.

Martínez, M. (1999). La nueva Ciencia: su desafío, lógica y método. México: Trillas.

Maturana, H. (1990) El árbol del conocimiento. Santiago: Universitaria

Melo, F. (1994). Desarrollo Organizacional. Enfoque integral. México: Limusa.

Peinado, H. y Rodríguez, J. (2007). Manual de Gestión y Administración Educativa. Bogotá: Magisterio

Pozo, J. (1997). Teoría Cognitiva del Aprendizaje. Madrid: Morata S.A.

Rodríguez, D. y Arnold, M. (1990). Sociedad y Teoría de Sistemas. Santiago: Universitaria.

Ruiz B., C. (1997). “Aprendizaje y Sistema Cognitivo Humano” UPEL-IPB. EDUCARE. 1(1), 73-90

Waztalvick, P., Beavín, J. y Jackson, D. (1995). Teoría de la Comunicación Humana. Barcelona: Hereder. 